\title{
Superconvergence for General Convex Optimal Control Problems Governed by Semilinear Parabolic Equations
}

\author{
Yongquan Dai ${ }^{1}$ and Yanping Chen ${ }^{2}$ \\ ${ }^{1}$ Hunan Key Laboratory for Computation and Simulation in Science and Engineering, Department of Mathematics, \\ Xiangtan University, Xiangtan, Hunan 411105, China \\ ${ }^{2}$ School of Mathematical Sciences, South China Normal University, Guangzhou, Guangdong 510631, China
}

Correspondence should be addressed to YanPing Chen; yanpingchen@scnu.edu.cn

Received 16 September 2013; Accepted 12 December 2013; Published 10 February 2014

Academic Editors: M. Braack, Y. M. Cheng, and W. Yeih

Copyright ( $\odot 2014$ Y. Dai and Y. Chen. This is an open access article distributed under the Creative Commons Attribution License, which permits unrestricted use, distribution, and reproduction in any medium, provided the original work is properly cited.

\begin{abstract}
We will investigate the superconvergence for the semidiscrete finite element approximation of distributed convex optimal control problems governed by semilinear parabolic equations. The state and costate are approximated by the piecewise linear functions and the control is approximated by piecewise constant functions. We present the superconvergence analysis for both the control variable and the state variables.
\end{abstract}

\section{Introduction}

Finite element approximation of optimal control problems plays a very important role in numerical methods for these problems. There have been extensive studies on this aspect, for example, [1-14]. A systematic introduction of finite element method for PDEs and optimal control problems can be found in, for example, [15-18]. The superconvergence of nonlinear parabolic problem was studied in [19]. In [20], superconvergence was obtained for parabolic optimal control problems with convex control constraints, where the state partial differential equations are linear.

Optimal control problems governed by nonlinear parabolic state equations, a priori error estimates of finite element approximation, were studied in, for example, [21, 22]. In this paper, we will study the superconvergence of both the control variable and the state variables for this problem.

The model optimal control problem that we shall study in detail is the following convex optimal control problem:

$$
\begin{aligned}
& \min _{u \in K}\left\{\int_{0}^{T}(g(y)+h(u)) d t\right\}, \\
& y_{t}(x, t)-\operatorname{div}(A(x) \nabla y(x, t))+\phi(y(x, t)) \\
& \quad=(f+B u)(x, t), \quad x \in \Omega, t \in(0, T],
\end{aligned}
$$

$$
\begin{gathered}
y(x, t)=0, \quad x \in \partial \Omega, t \in[0, T], \\
y(x, 0)=y_{0}(x), \quad x \in \Omega .
\end{gathered}
$$

Here, the bounded open set $\Omega \subset R^{2}$ is a convex polygon or has the smooth boundary $\partial \Omega$. Let $B$ be a linear continuous operator from $L^{2}\left(0, T ; L^{2}(\Omega)\right)$ to $L^{2}\left(0, T ; L^{2}(\Omega)\right)$ and $f \in$ $L^{2}\left(0, T ; L^{2}(\Omega)\right)$. Assume that $y_{0}(x) \in H^{2}(\Omega) \bigcap H_{0}^{1}(\Omega)$, $A(x)=\left(a_{i j}(x)\right)_{2 \times 2}$ with $a_{i j}(x) \in L^{\infty}(\Omega)$ being a symmetric matrix and, for any vector $X \in R^{2}$, there is a constant $c>0$ satisfying

$$
X^{t} A X \geq c\|X\|_{R^{2}}^{2} .
$$

Here, $K$ denotes the admissible set of the control variable, which is defined by

$$
\begin{gathered}
K=\left\{\tilde{u}(x, t) \in L^{2}\left(0, T ; L^{2}(\Omega)\right): \widetilde{u}(x, t) \geq 0,\right. \\
\text { a.e. } x \in \Omega, t \in[0, T]\} .
\end{gathered}
$$

In this paper, we adopt the standard notation $W^{m, p}(\Omega)$ for Sobolev spaces on $\Omega$ with a norm $\|\cdot\|_{m, p}$ given by

$$
\|v\|_{m, p}^{p}=\sum_{|\alpha| \leq m}\left\|D^{\alpha} v\right\|_{L^{p}(\Omega)}^{p}
$$


a semi-norm $|\cdot|_{m, p}$ given by

$$
|v|_{m, p}^{p}=\sum_{|\alpha|=m}\left\|D^{\alpha} v\right\|_{L^{p}(\Omega)}^{p} .
$$

We set $W_{0}^{m, p}(\Omega)=\left\{v \in W^{m, p}(\Omega):\left.v\right|_{\partial \Omega}=0\right\}$. For $p=2$, we denote

$$
\begin{array}{cc}
H^{m}(\Omega)=W^{m, 2}(\Omega), & H_{0}^{m}(\Omega)=W_{0}^{m, 2}(\Omega), \\
\|\cdot\|_{m}=\|\cdot\|_{m, 2}, & \|\cdot\|=\|\cdot\|_{0,2} .
\end{array}
$$

We denote by $L^{s}\left(J ; W^{m, p}(\Omega)\right)$ the Banach space of all $L^{s}$ integrable functions from $J$ into $W^{m, p}(\Omega)$, with norm

$$
\|\phi\|_{L^{s}\left(J ; W^{m, p}(\Omega)\right)}=\left(\int_{0}^{T}\|\phi\|_{W^{m, p}(\Omega)}^{s} d t\right)^{1 / s} \text { for } s \in[1, \infty),
$$

and the standard modification for $s=\infty$, where $J=[0, T]$. Similarly, one can define the spaces $H^{1}\left(0, T ; W^{m, q}(\Omega)\right)$ and $C^{k}\left(0, T ; W^{m, q}(\Omega)\right)$. The details can be found in [23]. In addition, $c$ and $C$ denote general positive constants independent of $h$.

The plan of the paper is as follows. In Section 2, we shall give a brief review on the finite element method and then construct the approximation scheme for the optimal control problem. In Section 3, we shall give some preliminaries and some intermediate error estimates. In Section 4, superconvergence results for both control and state variables were derived. In Section 5, we give a numerical example to demonstrate our theoretical results. In the last section we make a conclusion and state some future works.

\section{Approximation for the Optimal Control Problem}

To fix idea, we shall take the state space $W=L^{2}(0, T ; V)$ with $V=H_{0}^{1}(\Omega)$ and the control space $X=L^{2}(0, T ; U)$ with $U=$ $L^{2}(\Omega)$. Then the problem (1) can be restated as

$$
\begin{gathered}
\min _{u(t) \in K}\left\{\int_{0}^{T}(g(y)+h(u) d t)\right\}, \\
\left(y_{t}, w\right)+a(y, w)+(\phi(y), w)=(f+B u, w), \\
t \in(0, T], \quad \forall w \in V, \\
y(x, 0)=y_{0}(x),
\end{gathered}
$$

where $y \in H^{1}(0, T ; U) \bigcap W, u \in X$, and

$$
a(y, w)=\int_{\Omega}((A \nabla y) \cdot \nabla w), \quad \forall y, w \in V .
$$

It follows from the assumption on $A$ that there is a positive constant $C>0$ such that $a(v, v) \geq C\|v\|_{1}^{2}$, for all $v \in V$.

We make the following assumptions.

(1) $\phi(\cdot) \in W^{2, \infty}(-R, R)$ for any $R>0, \phi^{\prime}(y) \in L^{2}(\Omega)$ for any $y \in H^{1}(\Omega)$, and $\phi^{\prime} \geq 0$.
(2) Let $h(u)=\int_{\Omega} j(u)$, where $j(\cdot)$ is a smooth and convex function such that $j^{\prime \prime}(u) \in W^{1, \infty}(\Omega)$ and $j^{\prime \prime \prime}(\cdot) \in$ $L^{\infty}(R)$. The function $g(\cdot)$ has the same property as $h(\cdot)$.

It is well known (see, e.g., [24]) that the control problem (8)-(10) has a solution $(y, u)$ and that if a pair $(y, u)$ is the solution of (8)-(10), then there is a costate $p \in$ $H^{1}(0, T ; U) \bigcap W$ such that the triplet $(y, p, u)$ satisfies the following optimality conditions for $t \in(0, T]$ :

$$
\begin{gathered}
\left(y_{t}, w\right)+a(y, w)+(\phi(y), w)=(f+B u, w), \quad \forall w \in V \\
y(x, 0)=y_{0}(x), \quad x \in \Omega, \\
-\left(p_{t}, q\right)+a(q, p)+\left(\phi^{\prime}(y) p, q\right)=\left(g^{\prime}(y), q\right), \quad \forall q \in V \\
p(x, T)=0, \quad x \in \Omega, \\
\left(j^{\prime}(u)+B^{*} p, v-u\right) \geq 0, \quad \forall v \in K,
\end{gathered}
$$

where $B^{*}$ is the adjoint operator of $B$.

In the following we construct the finite element approximation for the optimal control problem (8)-(10). For ease of exposition we will assume that $\Omega$ is a convex polygon. Let $T_{h}$ be a quasi-uniform (in the sense of [25]) partition of $\Omega$ into triangles. And let $h$ be the maximum diameter of $T$ in $T_{h}$. Moreover, we set

$$
\begin{gathered}
U_{h}=\left\{\tilde{u} \in U:\left.\tilde{u}\right|_{T} \text { is constant on all } T \in T_{h}\right\}, \\
V_{h}=\left\{y_{h} \in C(\bar{\Omega}):\left.y_{h}\right|_{T} \in \mathbb{P}_{1}, \forall T \in T_{h},\left.y_{h}\right|_{\partial \Omega}=0\right\},
\end{gathered}
$$

where $\mathbb{P}_{1}$ is the space of polynomial of degree less than or equal to 1 .

For simplicity, in this paper we shall assume that $K_{h}=$ $K \cap U_{h}$. Now, the semidiscrete finite element approximation of the problem (8)-(10) is as follows:

$$
\begin{gathered}
\min _{u_{h}(t) \in K_{h}}\left\{\int_{0}^{T}\left(g\left(y_{h}\right)+h\left(u_{h}\right)\right) d t\right\}, \\
\left(\frac{\partial}{\partial t} y_{h}, w_{h}\right)+a\left(y_{h}, w_{h}\right)+\left(\phi\left(y_{h}\right), w_{h}\right)=\left(f+B u_{h}, w_{h}\right) \\
t \in(0, T], \quad \forall w_{h} \in V_{h}, \\
y_{h}(x, 0)=y_{0}^{h}(x),
\end{gathered}
$$

where $y_{h} \in H^{1}\left(0, T ; V_{h}\right)$ and $y_{0}^{h} \in V_{h}$ is an approximation of $y_{0}$ which will be defined below. The control problem (16)(18) has a solution $\left(y_{h}, u_{h}\right)$, and if a pair $\left(y_{h}, u_{h}\right)$ is the solution 
of (16)-(18), then there is a costate $p_{h}$ such that the triplet $\left(y_{h}, p_{h}, u_{h}\right)$ satisfies the following optimal conditions:

$$
\begin{array}{rr}
\left(\frac{\partial}{\partial t} y_{h}, w_{h}\right)+a\left(y_{h}, w_{h}\right)+\left(\phi\left(y_{h}\right), w_{h}\right)=\left(f+B u_{h}, w_{h}\right), \\
\forall w_{h} \in V_{h}, \\
y_{h}(x, 0)=y_{0}^{h}(x), \quad x \in \Omega,
\end{array}
$$

$$
\begin{gathered}
-\left(\frac{\partial}{\partial t} p_{h}, q_{h}\right)+a\left(q_{h}, p_{h}\right)+\left(\phi^{\prime}\left(y_{h}\right) p_{h}, q_{h}\right)=\left(g^{\prime}\left(y_{h}\right), q_{h}\right), \\
\forall q_{h} \in V_{h}, \\
p_{h}(x, T)=0, \quad x \in \Omega,
\end{gathered}
$$$$
\left(j^{\prime}\left(u_{h}\right)+B^{*} p_{h}, v_{h}-u_{h}\right) \geq 0, \quad \forall v_{h} \in K_{h} .
$$

\section{Some Preliminaries}

First, we shall use some intermediate variables. For any $\widetilde{u} \in K$, let $(y(\widetilde{u}), p(\widetilde{u}))$ be the solution of the following equations:

$$
\begin{gathered}
\left(y_{t}(\widetilde{u}), w\right)+a(y(\widetilde{u}), w)+(\phi(y(\widetilde{u})), w)=(f+B \widetilde{u}, w), \\
\forall w \in V, \\
y(\widetilde{u})(x, 0)=y_{0}(x), \quad x \in \Omega, \\
-\left(p_{t}(\widetilde{u}), q\right)+a(q, p(\widetilde{u}))+\left(\phi^{\prime}(y(\widetilde{u})) p(\widetilde{u}), q\right) \\
=\left(g^{\prime}(y(\widetilde{u})), q\right), \quad \forall q \in V, \\
p(\widetilde{u})(x, T)=0, \quad x \in \Omega .
\end{gathered}
$$$$
\forall w \in V
$$

Then, for any $\widetilde{u} \in K$, let $\left(y_{h}(\widetilde{u}), p_{h}(\widetilde{u})\right)$ be the solution of the following equations:

$$
\begin{gathered}
\left(\frac{\partial}{\partial t} y_{h}(\widetilde{u}), w_{h}\right)+a\left(y_{h}(\widetilde{u}), w_{h}\right)+\left(\phi\left(y_{h}(\widetilde{u})\right), w_{h}\right) \\
=\left(f+B \widetilde{u}, w_{h}\right), \quad \forall w_{h} \in V_{h}, \\
y_{h}(\widetilde{u})(x, 0)=y_{0}^{h}(x), \quad x \in \Omega, \\
-\left(\frac{\partial}{\partial t} p_{h}(\widetilde{u}), q_{h}\right)+a\left(q_{h}, p_{h}(\widetilde{u})\right)+\left(\phi^{\prime}\left(y_{h}(\widetilde{u})\right) p_{h}(\widetilde{u}), q_{h}\right) \\
=\left(g^{\prime}\left(y_{h}(\widetilde{u})\right), q_{h}\right), \quad \forall q_{h} \in V_{h}, \\
p_{h}(\widetilde{u})(x, T)=0, \quad x \in \Omega .
\end{gathered}
$$

Thus, we have

$$
(y, p)=(y(u), p(u)), \quad\left(y_{h}, p_{h}\right)=\left(y_{h}\left(u_{h}\right), p_{h}\left(u_{h}\right)\right) .
$$

We define the standard $L^{2}(\Omega)$-orthogonal projection $Q_{h}$ : $U \rightarrow U_{h}$, which satisfies, for any $\psi \in U$,

$$
\left(\psi-Q_{h} \psi, v_{h}\right)=0, \quad \forall v_{h} \in U_{h} .
$$

Next, let us recall the elliptic projection $R_{h}: V \rightarrow V_{h}$, which satisfies, for any $w \in V$,

$$
a\left(w-R_{h} w, w_{h}\right)=0, \quad \forall w_{h} \in V_{h} .
$$

Let

$$
y_{0}^{h}=R_{h} y_{0} .
$$

We have the approximation properties:

$$
\left\|\psi-Q_{h} \psi\right\|_{-s, r} \leq C h^{1+s}|\psi|_{1, r}, \quad s=0,1, \text { for } \psi \in W^{1, r}(\Omega),
$$

$$
\left\|w-R_{h} w\right\| \leq C h^{2}\|w\|_{2} \quad \text { for } w \in H^{2}(\Omega) \cap H_{0}^{1}(\Omega) .
$$

Now, we establish the following error estimates for the intermediate variables.

Lemma 1. Let $u$ be the solution of (12)-(14); for h sufficiently small, there exists a positive constant $C$ which only depends on $A$ and $\Omega$, such that

$$
\left\|y\left(Q_{h} u\right)-y(u)\right\|_{L^{\infty}\left(j ; L^{2}\right)}+\left\|y\left(Q_{h} u\right)-y(u)\right\|_{L^{2}\left(J ; H^{1}\right)} \leq C h^{2},
$$

$$
\left\|p\left(Q_{h} u\right)-p(u)\right\|_{L^{\infty}\left(j ; L^{2}\right)}+\left\|p\left(Q_{h} u\right)-p(u)\right\|_{L^{2}\left(J ; H^{1}\right)} \leq C h^{2} .
$$

Proof. Let $\widetilde{u}=Q_{h} u$ and $\widetilde{u}=u$ in (22)-(23), respectively; then we have the following error equations:

$$
\begin{aligned}
& \left(\frac{\partial}{\partial t}\left(y\left(Q_{h} u\right)-y(u)\right), w_{h}\right)+a\left(y\left(Q_{h} u\right)-y(u), w_{h}\right) \\
& \quad+\left(\phi\left(y\left(Q_{h} u\right)\right)-\phi(y(u)), w_{h}\right)=\left(B\left(Q_{h} u-u\right), w_{h}\right),
\end{aligned}
$$

$$
\begin{aligned}
- & \left.\frac{\partial}{\partial t}\left(p\left(Q_{h} u\right)-p(u)\right), q_{h}\right)+a\left(q_{h}, p\left(Q_{h} u\right)-p(u)\right) \\
& +\left(\phi^{\prime}\left(y\left(Q_{h} u\right)\right) p\left(Q_{h} u\right)-\phi^{\prime}(y(u)) p(u), q_{h}\right) \\
= & \left(g^{\prime}\left(y\left(Q_{h} u\right)\right)-g^{\prime}(y(u)), q_{h}\right),
\end{aligned}
$$

for any $w_{h} \in V_{h}$ and $q_{h} \in V_{h}$. We shall estimate (31) and (32), respectively.

First, let $w_{h}=y\left(Q_{h} u\right)-y(u)$ in (33); we have

$$
\begin{aligned}
\left(\frac{\partial}{\partial t}(\right. & \left.\left(y\left(Q_{h} u\right)-y(u)\right), y\left(Q_{h} u\right)-y(u)\right) \\
& +a\left(y\left(Q_{h} u\right)-y(u), y\left(Q_{h} u\right)-y(u)\right) \\
& +\left(\phi\left(y\left(Q_{h} u\right)\right)-\phi(y(u)), y\left(Q_{h} u\right)-y(u)\right) \\
= & \left(B\left(Q_{h} u-u\right), y\left(Q_{h} u\right)-y(u)\right) ;
\end{aligned}
$$


namely,

$$
\begin{aligned}
\frac{1}{2} \frac{d}{d t} & \left\|y\left(Q_{h} u\right)-y(u)\right\|^{2} \\
& +a\left(y\left(Q_{h} u\right)-y(u), y\left(Q_{h} u\right)-y(u)\right) \\
& +\left(\phi\left(y\left(Q_{h} u\right)\right)-\phi(y(u)), y\left(Q_{h} u\right)-y(u)\right) \\
= & \left(B\left(Q_{h} u-u\right), y\left(Q_{h} u\right)-y(u)\right) .
\end{aligned}
$$

Now, we estimate the right side of (36). Using the continuity of $B$ and (29), we have

$$
\begin{aligned}
& \left(B\left(Q_{h} u-u\right), y\left(Q_{h} u\right)-y(u)\right) \\
& \quad \leq C\left\|B^{*}\left(y\left(Q_{h} u\right)-y(u)\right)\right\|_{1}\left\|Q_{h} u-u\right\|_{-1} \\
& \quad \leq C h^{2}\left\|y\left(Q_{h} u\right)-y(u)\right\|_{1}\|u\|_{1} .
\end{aligned}
$$

Combining (36)-(37), using the $\epsilon$-Cauchy's inequality and the assumption of $A(x)$ and $\phi(\cdot)$, we have

$$
\begin{gathered}
\frac{1}{2} \frac{d}{d t}\left\|y\left(Q_{h} u\right)-y(u)\right\|^{2}+c\left\|y\left(Q_{h} u\right)-y(u)\right\|_{1}^{2} \\
\leq C h^{4}+\epsilon\left\|y\left(Q_{h} u\right)-y(u)\right\|_{1}^{2} .
\end{gathered}
$$

Notice that

$$
y\left(Q_{h} u\right)(x, 0)=y(u)(x, 0),
$$

then, integrating (38) in time and using Gronwall's lemma, we have

$$
\left\|y\left(Q_{h} u\right)-y(u)\right\|_{L^{\infty}\left(J ; L^{2}\right)}+\left\|y\left(Q_{h} u\right)-y(u)\right\|_{L^{2}\left(J ; H^{1}\right)} \leq C h^{2} .
$$

Then, by choosing $q_{h}=p\left(Q_{h} u\right)-p(u)$ in (34), we have

$$
\begin{gathered}
-\left(\frac{\partial}{\partial t}\left(p\left(Q_{h} u\right)-p(u)\right), p\left(Q_{h} u\right)-p(u)\right) \\
+a\left(p\left(Q_{h} u\right)-p(u), p\left(Q_{h} u\right)-p(u)\right) \\
+\left(\phi^{\prime}\left(y\left(Q_{h} u\right)\right) p\left(Q_{h} u\right)-\phi^{\prime}(y(u)) p(u),\right. \\
\left.p\left(Q_{h} u\right)-p(u)\right) \\
=\left(g^{\prime}\left(y\left(Q_{h} u\right)\right)-g^{\prime}(y(u)), p\left(Q_{h} u\right)-p(u)\right) ;
\end{gathered}
$$

namely,

$$
\begin{aligned}
-\frac{1}{2} & \frac{d}{d t}\left\|p\left(Q_{h} u\right)-p(u)\right\|^{2} \\
& +a\left(p\left(Q_{h} u\right)-p(u), p\left(Q_{h} u\right)-p(u)\right) \\
& +\left(\phi^{\prime}\left(y\left(Q_{h} u\right)\right)\left(p\left(Q_{h} u\right)-p(u)\right), p\left(Q_{h} u\right)-p(u)\right) \\
= & \left(g^{\prime}\left(y\left(Q_{h} u\right)\right)-g^{\prime}(y(u)), p\left(Q_{h} u\right)-p(u)\right) \\
& +\left(p(u)\left(\phi^{\prime}(y(u))-\phi^{\prime}\left(y\left(Q_{h} u\right)\right)\right), p\left(Q_{h} u\right)-p(u)\right) .
\end{aligned}
$$

Now, we estimate the right side of (42). From the assumption of the convex function $g(\cdot)$, we have

$$
\begin{aligned}
& \left(g^{\prime}\left(y\left(Q_{h} u\right)\right)-g^{\prime}(y(u)), p\left(Q_{h} u\right)-p(u)\right) \\
& \quad \leq C\|g\|_{W^{2, \infty}}\left\|y\left(Q_{h} u\right)-y(u)\right\| \cdot\left\|p\left(Q_{h} u\right)-p(u)\right\| \\
& \leq C\left\|p\left(Q_{h} u\right)-p(u)\right\|^{2}+C\left\|y\left(Q_{h} u\right)-y(u)\right\|^{2},
\end{aligned}
$$

and using the assumption of $\phi(\cdot)$ and $\epsilon$-Cauchy's inequality, we have

$$
\begin{aligned}
(p(u) & \left(\phi^{\prime}(y(u))-\phi^{\prime}\left(y\left(Q_{h} u\right)\right), p\left(Q_{h} u\right)-p(u)\right) \\
\leq & C\|p(u)\|_{0,4}\left\|\phi^{\prime}(y(u))-\phi^{\prime}\left(y\left(Q_{h} u\right)\right)\right\| \\
& \cdot\left\|p\left(Q_{h} u\right)-p(u)\right\|_{0,4} \\
\leq & C\|p(u)\|_{1} \cdot\|\phi\|_{W^{2, \infty}}\left\|y(u)-y\left(Q_{h} u\right)\right\| \\
& \cdot\left\|p\left(Q_{h} u\right)-p(u)\right\|_{1} \\
\leq & \left.C\left\|y\left(Q_{h} u\right)-y(u)\right\|^{2}+\epsilon\left\|p\left(Q_{h} u\right)-p(u)\right\|_{1}^{2}\right),
\end{aligned}
$$

where we used the embedding $\|v\|_{0,4} \leq C\|v\|_{1}$. Combining (42)-(44) and from the assumption of $A(x)$ and $\phi(\cdot)$, we have

$$
\begin{aligned}
-\frac{1}{2} & \frac{d}{d t}\left\|p\left(Q_{h} u\right)-p(u)\right\|^{2}+c\left\|p\left(Q_{h} u\right)-p(u)\right\|_{1}^{2} \\
\leq & C\left(\left\|y\left(Q_{h} u\right)-y(u)\right\|^{2}+\left\|p\left(Q_{h} u\right)-p(u)\right\|^{2}\right) \\
& +\epsilon\left\|p\left(Q_{h} u\right)-p(u)\right\|_{1}^{2} .
\end{aligned}
$$

Notice that

$$
p\left(Q_{h} u\right)(x, T)=p(u)(x, T) ;
$$

then integrating (45) in time, using Gronwall's lemma and (31), we have

$$
\left\|p\left(Q_{h} u\right)-p(u)\right\|_{L^{\infty}\left(j ; L^{2}\right)}+\left\|p\left(Q_{h} u\right)-p(u)\right\|_{L^{2}\left(j ; H^{1}\right)} \leq C h^{2},
$$

which completes the proof of Lemma 1.

Lemma 2. For any $\widetilde{u} \in K$, if the intermediate solution satisfies

$$
y(\widetilde{u}), p(\widetilde{u}) \in H^{1}\left(J ; H^{2}\right),
$$

then, one has

$$
\begin{aligned}
& \left\|y_{h}(\widetilde{\mathcal{u}})-R_{h} y(\widetilde{\mathcal{u}})\right\|_{L^{\infty}\left(j ; L^{2}\right)}+\left\|y_{h}(\widetilde{\mathcal{u}})-R_{h} y(\widetilde{u})\right\|_{L^{2}\left(J ; H^{1}\right)} \leq C h^{2}, \\
& \left\|p_{h}(\widetilde{\mathcal{u}})-R_{h} p(\widetilde{\mathcal{u}})\right\|_{L^{\infty}\left(j ; L^{2}\right)}+\left\|p_{h}(\widetilde{\mathcal{u}})-R_{h} p(\widetilde{\mathcal{u}})\right\|_{L^{2}\left(j ; H^{1}\right)} \leq C h^{2} .
\end{aligned}
$$


Proof. From (22)-(23) and (24), we have the following error equations:

$$
\begin{aligned}
& \left(\frac{\partial}{\partial t}\left(y_{h}(\widetilde{u})-y(\widetilde{u})\right), w_{h}\right)+a\left(y_{h}(\widetilde{u})-y(\widetilde{u}), w_{h}\right) \\
& \quad+\left(\phi\left(y_{h}(\widetilde{u})\right)-\phi(y(\widetilde{u})), w_{h}\right)=0, \\
& -\left(\frac{\partial}{\partial t}\left(p_{h}(\widetilde{u})-p(\widetilde{u})\right), q_{h}\right)+a\left(q_{h}, p_{h}(\widetilde{u})-p(\widetilde{u})\right) \\
& \quad+\left(\phi^{\prime}\left(y_{h}(\widetilde{u})\right) p_{h}(\widetilde{u})-\phi^{\prime}(y(\widetilde{u})) p(\widetilde{u}), q_{h}\right) \\
& =\left(g^{\prime}\left(y_{h}(\widetilde{u})\right)-g^{\prime}(y(\widetilde{u})), q_{h}\right)
\end{aligned}
$$

for any $w_{h} \in V_{h}$ and $q_{h} \in V_{h}$. Using the definition of $R_{h}$, the above equation can be restated as

$$
\begin{aligned}
\left(\frac{\partial}{\partial t}\left(y_{h}(\widetilde{u})-R_{h} y(\widetilde{u})\right), w_{h}\right)+a\left(y_{h}(\widetilde{u})-R_{h} y(\widetilde{u}), w_{h}\right) \\
\quad+\left(\phi\left(y_{h}(\widetilde{u})\right)-\phi\left(R_{h} y(\widetilde{u})\right), w_{h}\right) \\
=\left(\frac{\partial}{\partial t}\left(y(\widetilde{u})-R_{h} y(\widetilde{u})\right), w_{h}\right) \\
\quad+\left(\phi(y(\widetilde{u}))-\phi\left(R_{h} y(\widetilde{u})\right), w_{h}\right)
\end{aligned}
$$

$$
\begin{aligned}
& -\left(\frac{\partial}{\partial t}\left(p_{h}(\widetilde{u})-R_{h} p(\widetilde{u})\right), q_{h}\right)+a\left(q_{h}, p_{h}(\widetilde{u})-R_{h} p(\widetilde{u})\right) \\
& \quad+\left(\phi^{\prime}\left(y_{h}(\widetilde{u})\right)\left(p_{h}(\widetilde{u})-R_{h} p(\widetilde{u})\right), q_{h}\right) \\
& =\left(g^{\prime}\left(y_{h}(\widetilde{u})\right)-g^{\prime}(y(\widetilde{u})), q_{h}\right) \\
& \quad+\left(\frac{\partial}{\partial t}\left(R_{h} p(\widetilde{u})-p(\widetilde{u})\right), q_{h}\right) \\
& \quad+\left(p(\widetilde{u})\left(\phi^{\prime}(y(\widetilde{u}))-\phi^{\prime}\left(y_{h}(\widetilde{u})\right)\right), q_{h}\right) \\
& \quad+\left(\phi^{\prime}\left(y_{h}(\widetilde{u})\right)\left(p(\widetilde{u})-R_{h} p(\widetilde{u})\right), q_{h}\right) .
\end{aligned}
$$

Let $w_{h}=y_{h}(\widetilde{u})-R_{h} y(\widetilde{u})$ in (52); we have

$$
\begin{aligned}
\frac{1}{2} \frac{d}{d t} \| & y_{h}(\widetilde{u})-R_{h} y(\widetilde{u}) \|^{2} \\
& +a\left(y_{h}(\widetilde{u})-R_{h} y(\widetilde{u}), y_{h}(\widetilde{u})-R_{h} y(\widetilde{u})\right) \\
& +\left(\phi\left(y_{h}(\widetilde{u})\right)-\phi\left(R_{h} y(\widetilde{u})\right), y_{h}(\widetilde{u})-R_{h} y(\widetilde{u})\right) \\
= & \left(\frac{\partial}{\partial t}\left(y(\widetilde{u})-R_{h} y(\widetilde{u})\right), y_{h}(\widetilde{u})-R_{h} y(\widetilde{u})\right) \\
& +\left(\phi(y(\widetilde{u}))-\phi\left(R_{h} y(\widetilde{u})\right), y_{h}(\widetilde{u})-R_{h} y(\widetilde{u})\right) .
\end{aligned}
$$

For the first term of (54), using the definition of $R_{h}$, we have

$$
\begin{aligned}
& \left(\frac{\partial}{\partial t}\left(y(\widetilde{u})-R_{h} y(\widetilde{u})\right), y_{h}(\widetilde{u})-R_{h} y(\widetilde{u})\right) \\
& \quad=\left(\left(y_{t}(\widetilde{u})-R_{h} y_{t}(\widetilde{u})\right), y_{h}(\widetilde{u})-R_{h} y(\widetilde{u})\right) \\
& \quad \leq C h^{2}\left\|y_{t}(\widetilde{u})\right\|_{2}\left\|y_{h}(\widetilde{u})-R_{h} y(\widetilde{u})\right\| \\
& \quad \leq C h^{2}\left\|y_{h}(\widetilde{u})-R_{h} y(\widetilde{u})\right\| .
\end{aligned}
$$

Similarly, from the assumption of $\phi(\cdot)$, we can obtain

$$
\begin{aligned}
& \left(\phi(y(\widetilde{u}))-\phi\left(R_{h} y(\widetilde{u})\right), y_{h}(\widetilde{u})-R_{h} y(\widetilde{u})\right) \\
& \quad \leq C\|\phi\|_{1, \infty}\left\|y(\widetilde{u})-R_{h} y(\widetilde{u})\right\| \cdot\left\|y_{h}(\widetilde{u})-R_{h} y(\widetilde{u})\right\| \\
& \quad \leq C h^{2}\|\phi\|_{1, \infty}\|y(\widetilde{u})\|_{2}\left\|y_{h}(\widetilde{u})-R_{h} y(\widetilde{u})\right\| \\
& \quad \leq C h^{2}\left\|y_{h}(\widetilde{u})-R_{h} y(\widetilde{u})\right\| .
\end{aligned}
$$

Combining (54)-(56), using $\epsilon$-Cauchy's inequality, and from the assumption of $A(x)$ and $\phi$, we have

$$
\begin{gathered}
\frac{1}{2} \frac{d}{d t}\left\|y_{h}(\widetilde{u})-R_{h} y(\widetilde{u})\right\|^{2}+c\left\|y_{h}(\widetilde{u})-R_{h} y(\widetilde{u})\right\|_{1}^{2} \\
\leq C h^{4}+\frac{1}{2}\left\|y_{h}(\widetilde{u})-R_{h} y(\widetilde{u})\right\|^{2}
\end{gathered}
$$

Notice that

$$
y_{h}(\widetilde{u})(x, 0)-R_{h} y(\widetilde{u})(x, 0)=y_{0}^{h}-R_{h} y_{0}=0 .
$$

Then, integrating (57) in time and using Gronwall's lemma, we have

$$
\left\|y_{h}(\widetilde{u})-R_{h} y(\widetilde{u})\right\|_{L^{\infty}\left(J ; L^{2}\right)}+\left\|y_{h}(\widetilde{u})-R_{h} y(\widetilde{u})\right\|_{L^{2}\left(J ; H^{1}\right)} \leq C h^{2} .
$$

$$
\text { Let } q_{h}=p_{h}(\widetilde{u})-R_{h} p(\widetilde{u}) \text { in (53); we have }
$$

$$
\begin{aligned}
-\frac{1}{2} \| & p_{h}(\widetilde{u})-R_{h} p(\widetilde{u}) \|^{2} \\
& +a\left(p_{h}(\widetilde{u})-R_{h} p(\widetilde{u}), p_{h}(\widetilde{u})-R_{h} p(\widetilde{u})\right) \\
& +\left(\phi^{\prime}\left(y_{h}(\widetilde{u})\right)\left(p_{h}(\widetilde{u})-R_{h} p(\widetilde{u})\right), p_{h}(\widetilde{u})-R_{h} p(\widetilde{u})\right) \\
= & \left(g^{\prime}\left(y_{h}(\widetilde{u})\right)-g^{\prime}(y(\widetilde{u})), p_{h}(\widetilde{u})-R_{h} p(\widetilde{u})\right) \\
& +\left(\frac{\partial}{\partial t}\left(R_{h} p(\widetilde{u})-p(\widetilde{u})\right), p_{h}(\widetilde{u})-R_{h} p(\widetilde{u})\right) \\
& +\left(p(\widetilde{u})\left(\phi^{\prime}(y(\widetilde{u}))-\phi^{\prime}\left(y_{h}(\widetilde{u})\right)\right), p_{h}(\widetilde{u})-R_{h} p(\widetilde{u})\right) \\
& +\left(\phi^{\prime}\left(y_{h}(\widetilde{u})\right)\left(p(\widetilde{u})-R_{h} p(\widetilde{u})\right), p_{h}(\widetilde{u})-R_{h} p(\widetilde{u})\right) .
\end{aligned}
$$


Now we estimate the right side of (60):

$$
\begin{aligned}
& \left(g^{\prime}\left(y_{h}(\widetilde{u})\right)-g^{\prime}(y(\widetilde{u})), p_{h}(\widetilde{u})-R_{h} p(\widetilde{u})\right) \\
& \quad \leq C\|g\|_{W^{2, \infty}}\left\|y_{h}(\widetilde{u})-y(\widetilde{u})\right\| \cdot\left\|p_{h}(\widetilde{u})-R_{h} p(\widetilde{u})\right\| \\
& \quad \leq C h^{2}\|g\|_{W^{2, \infty}}\|y(\widetilde{u})\|_{2}\left\|p_{h}(\widetilde{u})-R_{h} p(\widetilde{u})\right\| \\
& \quad \leq C h^{2}\left\|p_{h}(\widetilde{u})-R_{h} p(\widetilde{u})\right\|,
\end{aligned}
$$

where we used the assumption of $g(\cdot)$ :

$$
\begin{aligned}
& \left(\frac{\partial}{\partial t}\left(R_{h} p(\tilde{u})-p(\tilde{u})\right), p_{h}(\tilde{u})-R_{h} p(\widetilde{u})\right) \\
& \quad \leq C\left\|R_{h} p_{t}(\widetilde{u})-p_{t}(\widetilde{u})\right\| \cdot\left\|p_{h}(\widetilde{u})-R_{h} p(\widetilde{u})\right\| \\
& \quad \leq C h^{2}\left\|p_{t}(\widetilde{u})\right\|_{2} \cdot\left\|p_{h}(\widetilde{u})-R_{h} p(\widetilde{u})\right\| \\
& \quad \leq C h^{2}\left\|p_{h}(\widetilde{u})-R_{h} p(\widetilde{u})\right\|
\end{aligned}
$$

where we used the definition of $R_{h}$ :

$$
\begin{aligned}
\left(p(\widetilde{u})\left(\phi^{\prime}(y(\widetilde{u}))-\phi^{\prime}\left(y_{h}(\widetilde{u})\right)\right), p_{h}(\widetilde{u})-R_{h} p(\widetilde{u})\right) \\
\leq C\|p(\widetilde{u})\|_{0,4}\left\|\phi^{\prime}(y(\widetilde{u}))-\phi^{\prime}\left(y_{h}(\widetilde{u})\right)\right\| \\
\quad \cdot\left\|p_{h}(\widetilde{u})-R_{h} p(\widetilde{u})\right\|_{0,4} \\
\leq C h^{2}\|p(\widetilde{u})\|_{1} \cdot\|\phi\|_{W^{2, \infty}}\|y(\widetilde{u})\|_{2} \cdot\left\|p_{h}(\widetilde{u})-R_{h} p(\widetilde{u})\right\|_{1} \\
\leq C h^{2}\left\|p_{h}(\widetilde{u})-R_{h} p(\widetilde{u})\right\|_{1},
\end{aligned}
$$

where we used the embedding $\|v\|_{0,4} \leq C\|v\|_{1}$ and the assumption of $\phi(\cdot)$ :

$$
\begin{aligned}
& \left(\phi^{\prime}\left(y_{h}(\widetilde{u})\right)\left(p(\widetilde{u})-R_{h} p(\widetilde{u})\right), p_{h}(\widetilde{u})-R_{h} p(\widetilde{u})\right) \\
& \quad \leq C\|\phi\|_{W^{1, \infty}}\left\|p(\widetilde{u})-R_{h} p(\widetilde{u})\right\| \cdot\left\|p_{h}(\widetilde{u})-R_{h} p(\widetilde{u})\right\| \\
& \quad \leq C h^{2}\|\phi\|_{W^{1, \infty}}\|p(\widetilde{u})\|_{2} \cdot\left\|p_{h}(\widetilde{u})-R_{h} p(\widetilde{u})\right\| \\
& \quad \leq C h^{2}\left\|p_{h}(\widetilde{u})-R_{h} p(\widetilde{u})\right\|,
\end{aligned}
$$

where we used the assumption of $\phi(\cdot)$ and the definition of $R_{h}$.

Notice that

$$
p_{h}(\widetilde{u})(x, T)=R_{h} p(\widetilde{u})(x, T)=0,
$$

then, combining (60)-(64), using $\epsilon$-Cauchy's inequality, and the assumption of $A(x)$ and $\phi(\cdot)$, we have

$$
\begin{aligned}
& -\frac{1}{2} \frac{d}{d t}\left\|p_{h}(\tilde{u})-R_{h} p(\widetilde{u})\right\|^{2}+c\left\|p_{h}(\widetilde{u})-R_{h} p(\widetilde{u})\right\|_{1}^{2} \\
& \quad \leq C h^{4}+\frac{1}{2}\left\|p_{h}(\widetilde{u})-R_{h} p(\widetilde{u})\right\|^{2}+\epsilon\left\|p_{h}(\widetilde{u})-R_{h} p(\widetilde{u})\right\|_{1}^{2} .
\end{aligned}
$$

Integrating (66) in time and using Gronwall lemma, we have

$$
\left\|p_{h}(\widetilde{u})-R_{h} p(\widetilde{u})\right\|_{L^{\infty}\left(J ; L^{2}\right)}+\left\|p_{h}(\widetilde{u})-R_{h} p(\widetilde{u})\right\|_{L^{2}\left(J ; H^{1}\right)} \leq C h^{2},
$$

which implies (50). Thus, we complete the proof of Lemma 2.

\section{Superconvergence Properties}

In this section, we will discuss the superconvergence for both the control variable and the state variables by using the results we have got before. Let $\pi^{c}$ be the average operator defined in [26]. Let

$$
\begin{gathered}
\Omega^{+}=\left\{\cup \tau: \tau \subset \Omega,\left.u\right|_{\tau}>0\right\}, \\
\Omega^{0}=\left\{\cup \tau: \tau \subset \Omega,\left.u\right|_{\tau}=0\right\}, \\
\Omega^{b}=\Omega \backslash\left(\Omega^{+} \cup \Omega^{0}\right) .
\end{gathered}
$$

In this paper, we assume that $u$ and $T_{h}$ are regular such that meas $\left(\Omega^{b}\right) \leq C h$.

Let $y(u)$ and $y_{h}\left(u_{h}\right)$ be the solution of (9) and (17), respectively. Set

$$
\begin{gathered}
J(u)=\left\{\int_{0}^{T}(g(y)+h(u)) d t\right\}, \\
J_{h}\left(u_{h}\right)=\left\{\int_{0}^{T}\left(g\left(y_{h}\left(u_{h}\right)\right)+h\left(u_{h}\right)\right) d t\right\} .
\end{gathered}
$$

Then the reduced problems of (8) and (16) read as

$$
\begin{gathered}
\min _{u \in K}\{J(u)\}, \\
\min _{u_{h} \in K_{h}}\left\{J_{h}\left(u_{h}\right)\right\},
\end{gathered}
$$

respectively. It can be shown that

$$
\begin{gathered}
\left(J^{\prime}(u), v\right)=\int_{0}^{T}\left(j^{\prime}(u)+B^{*} p, v\right) d t \\
\left(J^{\prime}\left(u_{h}\right), v\right)=\int_{0}^{T}\left(j^{\prime}\left(u_{h}\right)+B^{*} p\left(u_{h}\right), v\right) d t \\
\left(J^{\prime}\left(Q_{h} u\right), v\right)=\int_{0}^{T}\left(j^{\prime}\left(Q_{h} u\right)+B^{*} p\left(Q_{h} u\right), v\right) d t, \\
\left(J_{h}^{\prime}\left(u_{h}\right), v\right)=\int_{0}^{T}\left(j^{\prime}\left(u_{h}\right)+B^{*} p_{h}, v\right) d t,
\end{gathered}
$$

where $p\left(Q_{h} u\right)$ is the solution of (22)-(23) with $\tilde{u}=Q_{h} u$.

In many applications, $J(\cdot)$ is uniform convex near the solution $u$. The convexity of $J(\cdot)$ is closely related to the second order sufficient conditions of the control problem, which are assumed in many studies on numerical methods of the problem. For instance, in some applications, $u \rightarrow g(y(u))$ is convex; see [27] for examples. Thus if $j(\cdot)$ is uniform convex 
(e.g., $j(u)=\int_{\Omega U} u^{2}$ ), which is frequently met, then, there is a constant $c>0$, independent of $h$, such that

$$
\left(J^{\prime}\left(Q_{h} u\right)-J^{\prime}\left(u_{h}\right), Q_{h} u-u_{h}\right) \geq c\left\|Q_{h} u-u_{h}\right\|_{L^{2}\left(J ; L^{2}\right)}^{2},
$$

where $u$ and $u_{h}$ are solutions of (70) and (71), respectively, $Q_{h} u$ is the orthogonal projection of $u$ which is defined in (26). We shall assume the above inequality throughout this paper.

First, we are going to formulate the superconvergence result for the control variable.

Theorem 3. Let $u$ be the solution of (12)-(14) and let $u_{h}$ be the solution of (19)-(21). One assumes that the exact control and state solution satisfy

$$
\begin{gathered}
u, j^{\prime}(u)+B^{*} p \in L^{2}\left(J ; W^{1, \infty}(\Omega)\right), \\
y(u), p(u) \in H^{1}\left(J ; H^{2}\right) .
\end{gathered}
$$

Then, one has

$$
\left\|Q_{h} u-u_{h}\right\|_{L^{2}\left(J ; L^{2}\right)} \leq C h^{3 / 2}
$$

Proof. Let $v=u_{h}$ in (14) and $v_{h}=Q_{h} u$ in (21) and then, add the two inequalities; we have

$$
\begin{aligned}
& \left(j^{\prime}\left(u_{h}\right)+B^{*} p_{h}-j^{\prime}(u)-B^{*} p, Q_{h} u-u_{h}\right) \\
& \quad+\left(j^{\prime}(u)+B^{*} p, Q_{h} u-u\right) \geq 0 .
\end{aligned}
$$

Hence,

$$
\begin{aligned}
& \left(j^{\prime}\left(Q_{h} u\right)-j^{\prime}\left(u_{h}\right), Q_{h} u-u_{h}\right) \\
& \quad \leq\left(j^{\prime}\left(Q_{h} u\right)-j^{\prime}(u), Q_{h} u-u_{h}\right) \\
& \quad+\left(B^{*} p_{h}-B^{*} p, Q_{h} u-u_{h}\right)+\left(j^{\prime}(u)+B^{*} p, Q_{h} u-u\right) .
\end{aligned}
$$

For the second term of the right hand of (77), we divide it into four parts:

$$
\begin{aligned}
\left(B^{*} p_{h}-B^{*} p, Q_{h} u-u_{h}\right) \\
=\left(B^{*} p_{h}-B^{*} R_{h} p\left(u_{h}\right), Q_{h} u-u_{h}\right) \\
\quad+\left(B^{*} R_{h} p\left(u_{h}\right)-B^{*} p\left(u_{h}\right), Q_{h} u-u_{h}\right) \\
\quad+\left(B^{*} p\left(u_{h}\right)-B^{*} p\left(Q_{h} u\right), Q_{h} u-u_{h}\right) \\
\quad+\left(B^{*} p\left(Q_{h} u\right)-B^{*} p, Q_{h} u-u_{h}\right),
\end{aligned}
$$

then, from (77)-(78), we have

$$
\begin{aligned}
\left(j^{\prime}\right. & \left.\left(Q_{h} u\right)-j^{\prime}\left(u_{h}\right), Q_{h} u-u_{h}\right) \\
& -\left(B^{*} p\left(u_{h}\right)-B^{*} p\left(Q_{h} u\right), Q_{h} u-u_{h}\right) \\
\leq & \left(j^{\prime}\left(Q_{h} u\right)-j^{\prime}(u), Q_{h} u-u_{h}\right) \\
& +\left(B^{*} p_{h}-B^{*} R_{h} p\left(u_{h}\right), Q_{h} u-u_{h}\right) \\
& +\left(B^{*} R_{h} p\left(u_{h}\right)-B^{*} p\left(u_{h}\right), Q_{h} u-u_{h}\right) \\
& +\left(B^{*} p\left(Q_{h} u\right)-B^{*} p, Q_{h} u-u_{h}\right) \\
& +\left(j^{\prime}(u)+B^{*} p, Q_{h} u-u\right) .
\end{aligned}
$$

Using the definition of $R_{h}$ and the assumption of $B$, we have

$$
\begin{aligned}
& \left(B^{*} R_{h} p\left(u_{h}\right)-B^{*} p\left(u_{h}\right), Q_{h} u-u_{h}\right) \\
& \quad \leq C\left\|R_{h} p\left(u_{h}\right)-p\left(u_{h}\right)\right\| \cdot\left\|Q_{h} u-u_{h}\right\| \\
& \quad \leq C h^{2}\left\|p\left(u_{h}\right)\right\|_{2} \cdot\left\|Q_{h} u-u_{h}\right\| \\
& \quad \leq C h^{2}\left\|Q_{h} u-u_{h}\right\| .
\end{aligned}
$$

From Taylor's expansion of the function $j(\cdot)$, there exists some value $0 \leq \theta \leq 1$ such that

$$
\begin{aligned}
\left(j^{\prime}\left(Q_{h} u\right)-j^{\prime}(u), Q_{h} u-u_{h}\right) \\
=\left(j^{\prime \prime}(u)\left(Q_{h} u-u\right)\right. \\
\left.\quad+\frac{1}{2} j^{\prime \prime \prime}\left(u+\theta\left(Q_{h} u-u\right)\right)\left(Q_{h} u-u\right)^{2}, Q_{h} u-u_{h}\right) \\
=\left(\left(j^{\prime \prime}(u)-\pi^{c}\left(j^{\prime \prime}(u)\right)\right) \cdot\left(Q_{h} u-u\right), Q_{h} u-u_{h}\right) \\
\quad+\left(\frac{1}{2} j^{\prime \prime \prime}\left(u+\theta\left(Q_{h} u-u\right)\right)\left(Q_{h} u-u\right)^{2}, Q_{h} u-u_{h}\right) \\
\leq C h\left\|j^{\prime \prime}\right\|_{1, \infty}\left\|Q_{h} u-u\right\| \cdot\left\|Q_{h} u-u_{h}\right\| \\
\quad+\frac{1}{2} C\left\|j^{\prime \prime \prime}(\cdot)\right\|_{0, \infty}\left\|Q_{h} u-u\right\|_{0,4}^{2} \cdot\left\|Q_{h} u-u_{h}\right\| \\
\leq C h^{2}\left\|Q_{h} u-u_{h}\right\|,
\end{aligned}
$$

where we used the assumption of $j(\cdot)$ and the approximation property (29).

Notice that

$$
\begin{aligned}
& \left(j^{\prime}(u)+B^{*} p, Q_{h} u-u\right) \\
& \quad=\int_{\Omega^{+}}+\int_{\Omega^{0}}+\int_{\Omega^{b}}\left(j^{\prime}(u)+B^{*} p, Q_{h} u-u\right) d x .
\end{aligned}
$$

Obviously, $\left.\left(Q_{h} u-u\right)\right|_{\Omega^{0}}=0$. From (14), we have pointwise a.e. $\left(j^{\prime}(u)+B^{*} p\right) \geq 0$; we choose $\left.\widetilde{u}\right|_{\Omega^{+}}=0$ and $\left.\widetilde{u}\right|_{\Omega \backslash \Omega^{+}}=u$, 
so that $\left.\left(j^{\prime}(u)+B^{*} p, u\right)\right|_{\Omega^{+}} \leq 0$. Hence, $\left.\left(j^{\prime}(u)+B^{*} p\right)\right|_{\Omega^{+}}=0$. Then,

$$
\begin{aligned}
& \left(j^{\prime}(u)+B^{*} p, Q_{h} u-u\right) \\
& \quad=\left(j^{\prime}(u)+B^{*} p, Q_{h} u-u\right)_{\Omega^{b}} \\
& \quad \leq\left(j^{\prime}(u)+B^{*} p-\pi^{c}\left(j^{\prime}(u)+B^{*} p\right), Q_{h} u-u\right)_{\Omega^{b}} \\
& \quad \leq C h^{2}\left\|j^{\prime}(u)+B^{*} p\right\|_{1, \Omega^{b}}\|u\|_{1, \Omega^{b}} \\
& \quad \leq C h^{2}\left\|j^{\prime}(u)+B^{*} p\right\|_{1, \infty}\|u\|_{1, \infty} \operatorname{meas}\left(\Omega^{b}\right) \\
& \leq C h^{3} .
\end{aligned}
$$

From the assumption of $J(\cdot)$, we have

$$
\begin{aligned}
& \int_{0}^{T}\left(j^{\prime}\left(Q_{h} u\right)-j^{\prime}\left(u_{h}\right), Q_{h} u-u_{h}\right) d t \\
& \quad-\int_{0}^{T}\left(B^{*} p\left(u_{h}\right)-B^{*} p\left(Q_{h} u\right), Q_{h} u-u_{h}\right) d t \\
& =\int_{0}^{T}\left(j^{\prime}\left(Q_{h} u\right)+B^{*} p\left(Q_{h} u\right), Q_{h} u-u_{h}\right) d t \\
& \quad-\int_{0}^{T}\left(j^{\prime}\left(u_{h}\right)+B^{*} p\left(u_{h}\right), Q_{h} u-u_{h}\right) d t \\
& =\left(J^{\prime}\left(Q_{h} u\right)-J^{\prime}\left(u_{h}\right), Q_{h} u-u_{h}\right) \\
& \geq c\left\|Q_{h} u-u_{h}\right\|_{L^{2}\left(J ; L^{2}\right)}^{2} .
\end{aligned}
$$

Then, integrating (79) in time and combining Lemmas 1 and 2 and (79)-(84), we have

$$
c\left\|Q_{h} u-u_{h}\right\|_{L^{2}\left(J ; L^{2}\right)}^{2} \leq C h^{3}+\epsilon\left\|Q_{h} u-u_{h}\right\|_{L^{2}\left(j ; L^{2}\right)}^{2},
$$

where we have used $\epsilon$-Cauchy's inequality which implies (75). Thus, we complete the proof of Theorem 3.

In the following, we shall establish the superconvergence results for the state variable $y$ and costate variable $p$.

Theorem 4. Let $u$ be the solution of (12)-(14) and let $u_{h}$ be the solution of (19)-(21). One assumes that the exact control and state solution satisfy

$$
\begin{gathered}
u, j^{\prime}(u)+B^{*} p \in L^{2}\left(J ; W^{1, \infty}(\Omega)\right), \\
y(u), p(u) \in H^{1}\left(J ; H^{2}\right) .
\end{gathered}
$$

Then, one has

$$
\begin{aligned}
& \left\|y_{h}-R_{h} y\right\|_{L^{\infty}\left(J ; L^{2}\right)}+\left\|y_{h}-R_{h} y\right\|_{L^{2}\left(J ; H^{1}\right)} \leq C h^{3 / 2}, \\
& \left\|p_{h}-R_{h} p\right\|_{L^{\infty}\left(J ; L^{2}\right)}+\left\|p_{h}-R_{h} p\right\|_{L^{2}\left(J ; H^{1}\right)} \leq C h^{3 / 2} .
\end{aligned}
$$

Proof. First, we have the following error equation from (12) and (19):

$$
\begin{aligned}
& \left(\frac{\partial}{\partial t}\left(y_{h}-y\right), w_{h}\right)+a\left(y_{h}-y, w_{h}\right)+\left(\phi\left(y_{h}\right)-\phi(y), w_{h}\right) \\
& \quad=\left(B\left(u_{h}-u\right), w_{h}\right)
\end{aligned}
$$

for any $w_{h} \in V_{h}$.

Using the definition of $R_{h}$ in (27), we have

$$
\begin{aligned}
\left(\frac{\partial}{\partial t}\left(y_{h}-R_{h} y\right), w_{h}\right)+a\left(y_{h}-R_{h} y, w_{h}\right) \\
\quad+\left(\phi\left(y_{h}\right)-\phi\left(R_{h} y\right), w_{h}\right) \\
=\left(B\left(u_{h}-u\right), w_{h}\right)+\left(\frac{\partial}{\partial t}\left(y-R_{h} y\right), w_{h}\right) \\
\quad+\left(\phi(y)-\phi\left(R_{h} y\right), w_{h}\right)
\end{aligned}
$$

for any $w_{h} \in V_{h}$.

We take $w_{h}=y_{h}-R_{h} y$ in (90), and using the assumption of $A(x)$ and $\phi(\cdot)$, then

$$
\begin{aligned}
\frac{1}{2} \frac{d}{d t} & \left\|y_{h}-R_{h} y\right\|^{2}+c\left\|y_{h}-R_{h} y\right\|_{1}^{2} \\
\leq & \left(\frac{\partial}{\partial t}\left(y_{h}-R_{h} y\right), y_{h}-R_{h} y\right)+a\left(y_{h}-R_{h} y, y_{h}-R_{h} y\right) \\
& +\left(\phi\left(y_{h}\right)-\phi\left(R_{h} y\right), y_{h}-R_{h} y\right) \\
= & \left(B\left(u_{h}-u\right), y_{h}-R_{h} y\right)+\left(y_{t}-R_{h} y_{t}, y_{h}-R_{h} y\right) \\
& +\left(\phi(y)-\phi\left(R_{h} y\right), y_{h}-R_{h} y\right) .
\end{aligned}
$$

Now, we estimate the right hand of (91). From (29)-(30), (75), and using $\epsilon$-Cauchy's inequality, we have

$$
\begin{aligned}
(B & \left.\left(u_{h}-u\right), y_{h}-R_{h} y\right) \\
= & \left(B\left(u_{h}-Q_{h} u\right), y_{h}-R_{h} y\right)+\left(B\left(Q_{h} u-u\right), y_{h}-R_{h} y\right) \\
\leq & C\left\|u_{h}-Q_{h} u\right\| \cdot\left\|y_{h}-R_{h} y\right\| \\
& +C\left\|Q_{h} u-u\right\|_{-1}\left\|B^{*}\left(y_{h}-R_{h} y\right)\right\|_{1} \\
\leq & C\left\|u_{h}-Q_{h} u\right\| \cdot\left\|y_{h}-R_{h} y\right\|+C h^{2}\|u\|_{1}\left\|y_{h}-R_{h} y\right\|_{1} \\
\leq & C\left(\left\|u_{h}-Q_{h} u\right\|^{2}+\left\|y_{h}-R_{h} y\right\|^{2}\right)+C h^{4}+\epsilon\left\|y_{h}-R_{h} y\right\|_{1}^{2},
\end{aligned}
$$

$$
\left(y_{t}-R_{h} y_{t}, y_{h}-R_{h} y\right)
$$$$
\leq C\left\|y_{t}-R_{h} y_{t}\right\| \cdot\left\|y_{h}-R_{h} y\right\|
$$$$
\leq C h^{2}\left\|y_{t}\right\|_{2}\left\|y_{h}-R_{h} y\right\|
$$

$\leq C h^{4}+C\left\|y_{h}-R_{h} y\right\|^{2}$. 
Then, using the assumption of $\phi(\cdot)$, we have

$$
\begin{aligned}
& \left(\phi(y)-\phi\left(R_{h} y\right), y_{h}-R_{h} y\right) \\
& \quad \leq C\|\phi\|_{1, \infty}\left\|y-R_{h} y\right\| \cdot\left\|y_{h}-R_{h} y\right\| \\
& \leq C h^{2}\|\phi\|_{1, \infty}\|y\|_{2} \cdot\left\|y_{h}-R_{h} y\right\| \\
& \quad \leq C h^{4}+C\left\|y_{h}-R_{h} y\right\|^{2} .
\end{aligned}
$$

Therefore, inserting (92)-(94) in (91), we have

$$
\begin{aligned}
& \frac{1}{2} \frac{d}{d t}\left\|y_{h}-R_{h} y\right\|^{2}+c\left\|y_{h}-R_{h} y\right\|_{1}^{2} \\
& \quad \leq C\left\|u_{h}-Q_{h} u\right\|^{2}+C\left\|y_{h}-R_{h} y\right\|^{2}+C h^{4} .
\end{aligned}
$$

Notice that

$$
y_{h}(x, 0)-R_{h} y(x, 0)=0
$$

then, integrating (95) in time, using Gronwall's lemma, and from the result of Theorem 3 , we can easily obtain that

$$
\left\|y_{h}-R_{h} y\right\|_{L^{\infty}\left(J ; L^{2}\right)}+\left\|y_{h}-R_{h} y\right\|_{L^{2}\left(J ; H^{1}\right)} \leq C h^{3 / 2}
$$

which implies (87).

Then, from (13) and (20), we have the following error equation

$$
\begin{aligned}
& -\left(\frac{\partial}{\partial t}\left(p_{h}-p\right), q_{h}\right)+a\left(q_{h}, p_{h}-p\right) \\
& \quad+\left(\phi^{\prime}\left(y_{h}\right) p_{h}-\phi^{\prime}(y) p, q_{h}\right)=\left(g^{\prime}\left(y_{h}\right)-g^{\prime}(y), q_{h}\right),
\end{aligned}
$$

for any $q_{h} \in V_{h}$. Using the definition of $R_{h}$ in (27), we have

$$
\begin{aligned}
& -\left(\frac{\partial}{\partial t}\left(p_{h}-R_{h} p\right), q_{h}\right)+a\left(q_{h}, p_{h}-R_{h} p\right) \\
& \quad+\left(\phi^{\prime}\left(y_{h}\right)\left(p_{h}-R_{h} p\right), q_{h}\right) \\
& =\left(g^{\prime}\left(y_{h}\right)-g^{\prime}(y), q_{h}\right)+\left(\frac{\partial}{\partial t}\left(R_{h} p-p\right), q_{h}\right) \\
& \quad+\left(\phi^{\prime}\left(y_{h}\right)\left(p-R_{h} p\right), q_{h}\right) \\
& \quad+\left(p\left(\phi^{\prime}(y)-\phi^{\prime}\left(y_{h}\right)\right), q_{h}\right) .
\end{aligned}
$$

We take $q_{h}=p_{h}-R_{h} p$, and using the assumption of $\phi(\cdot)$ and $A(x)$, then

$$
\begin{aligned}
&-\frac{1}{2} \frac{d}{d t}\left\|p_{h}-R_{h} p\right\|^{2}+c\left\|p_{h}-R_{h} p\right\|_{1}^{2} \\
& \leq-\left(\frac{\partial}{\partial t}\left(p_{h}-R_{h} p\right), p_{h}-R_{h} p\right) \\
&+a\left(p_{h}-R_{h} p, p_{h}-R_{h} p\right) \\
&+\left(\phi^{\prime}\left(y_{h}\right)\left(p_{h}-R_{h} p\right), p_{h}-R_{h} p\right) \\
&=\left(g^{\prime}\left(y_{h}\right)-g^{\prime}(y), p_{h}-R_{h} p\right) \\
&+\left(R_{h} p_{t}-p_{t}, p_{h}-R_{h} p\right) \\
&+\left(\phi^{\prime}\left(y_{h}\right)\left(p-R_{h} p\right), p_{h}-R_{h} p\right) \\
&+\left(p\left(\phi^{\prime}(y)-\phi^{\prime}\left(y_{h}\right)\right), p_{h}-R_{h} p\right) .
\end{aligned}
$$

Now, we estimate the right hand of (100). From the assumption of $g$, we have

$$
\begin{aligned}
& \left(g^{\prime}\left(y_{h}\right)-g^{\prime}(y), p_{h}-R_{h} p\right) \\
& =\left(g^{\prime}\left(y_{h}\right)-g^{\prime}\left(R_{h} y\right), p_{h}-R_{h} p\right) \\
& \quad+\left(g^{\prime}\left(R_{h} y\right)-g^{\prime}(y), p_{h}-R_{h} p\right) \\
& \leq C\|g\|_{W^{2, \infty}}\left(\left\|y_{h}-R_{h} y\right\| \cdot\left\|p_{h}-R_{h} p\right\|\right. \\
& \left.\quad+\left\|R_{h} y-y\right\| \cdot\left\|p_{h}-R_{h} p\right\|\right) \\
& \leq C\left\|y_{h}-R_{h} y\right\| \cdot\left\|p_{h}-R_{h} p\right\|+C h^{2}\|y\|_{2} \cdot\left\|p_{h}-R_{h} p\right\| \\
& \leq C h^{4}+C\left\|y_{h}-R_{h} y\right\|^{2}+C\left\|p_{h}-R_{h} p\right\|^{2} .
\end{aligned}
$$

Using the definition of $R_{h}$, we have

$$
\begin{aligned}
& \left(p_{t}-R_{h} p_{t}, p_{h}-R_{h} p\right) \\
& \quad \leq C\left\|p_{t}-R_{h} p_{t}\right\| \cdot\left\|p_{h}-R_{h} p\right\| \\
& \leq C h^{2}\left\|p_{t}\right\|_{2}\left\|p_{h}-R_{h} p\right\| \\
& \leq C h^{4}+C\left\|p_{h}-R_{h} p\right\|^{2} .
\end{aligned}
$$

From the assumption of $\phi(\cdot)$ and the definition of $R_{h}$, we can obtain

$$
\begin{aligned}
& \left(\phi^{\prime}\left(y_{h}\right)\left(p-R_{h} p\right), p_{h}-R_{h} p\right) \\
& \leq C\|\phi\|_{W^{1, \infty}}\left\|p-R_{h} p\right\| \cdot\left\|p_{h}-R_{h} p\right\| \\
& \leq C h^{2}\|\phi\|_{W^{1, \infty}}\|p\|_{2} \cdot\left\|p_{h}-R_{h} p\right\| \\
& \leq C h^{4}+C\left\|p_{h}-R_{h} p\right\|^{2} .
\end{aligned}
$$


From the assumption of $\phi(\cdot)$ and $\epsilon$-Cauchy's inequality, we have

$$
\begin{aligned}
& \left(p\left(\phi^{\prime}(y)-\phi^{\prime}\left(y_{h}\right)\right), p_{h}-R_{h} p\right) \\
& \quad \leq C\|p\|_{0,4}\left\|\phi^{\prime}(y)-\phi^{\prime}\left(y_{h}\right)\right\| \cdot\left\|p_{h}-R_{h} p\right\|_{0,4} \\
& \quad \leq C\|p\|_{1} \cdot\|\phi\|_{W^{2, \infty}}\left\|y-y_{h}\right\| \cdot\left\|p_{h}-R_{h} p\right\|_{1} \\
& \quad \leq C\left(\left\|y-R_{h} y\right\|+\left\|R_{h} y-y_{h}\right\|\right)\left\|p_{h}-R_{h} p\right\|_{1} \\
& \quad \leq C h^{2}\|y\|_{2}\left\|p_{h}-R_{h} p\right\|_{1}+C\left\|y_{h}-R_{h} y\right\|\left\|p_{h}-R_{h} p\right\|_{1} \\
& \quad \leq C h^{4}+C\left\|y_{h}-R_{h} y\right\|^{2}+\epsilon\left\|p_{h}-R_{h} p\right\|_{1}^{2} .
\end{aligned}
$$

Therefore, inserting (101)-(104) in (100), we have

$$
\begin{aligned}
- & \frac{1}{2} \frac{d}{d t}\left\|p_{h}-R_{h} p\right\|^{2}+c\left\|p_{h}-R_{h} p\right\|_{1}^{2} \\
& \leq C\left\|y_{h}-R_{h} y\right\|^{2}+C\left\|p_{h}-R_{h} p\right\|^{2}+\epsilon\left\|p_{h}-R_{h} p\right\|_{1}^{2}+C h^{4} .
\end{aligned}
$$

Notice that

$$
p_{h}(x, T)-R_{h} p(x, T)=0,
$$

then, integrating (105) in time, using Gronwall's lemma and (97), we have

$$
\left\|p_{h}-R_{h} p\right\|_{L^{\infty}\left(j ; L^{2}\right)}+\left\|p_{h}-R_{h} p\right\|_{L^{2}\left(j ; H^{1}\right)} \leq C h^{3 / 2}
$$

Thus, we complete the proof of Theorem 4 .

\section{Numerical Example}

In this section, we carry out a numerical example to demonstrate our theoretical results. The optimal problem was solved numerically by a precondition projection algorithm; see, for instance, [28], with codes developed based on AFEPack [29]. In order to validate the superconvergence results, we shall consider the following full-discrete scheme. Let $t_{i}:=i \Delta t, i=$ $0,1, \ldots, N, \Delta t>0$ being the time-step, and $N:=[T / \Delta t]$, the integral part of $T / \Delta t$. In the example, we choose the domain $\Omega=[0,1] \times[0,1]$ and $B=I$.

We now shall consider the fully discrete approximation for semidiscrete problem (19)-(21) by using the backward
Euler scheme in time. The scheme is as follows: find $\left(y_{h}^{i}, p_{h}^{i}, u_{h}^{i}\right) \in V_{h} \times V_{h} \times K_{h}$ such that

$$
\begin{aligned}
& \left(\frac{y_{h}^{i}-y_{h}^{i-1}}{\Delta t}, w_{h}\right)+a\left(y_{h}^{i}, w_{h}\right)+\left(\phi\left(y_{h}^{i}\right), w_{h}\right) \\
& =\left(f\left(x, t_{i}\right)+B u_{h}^{i}, w_{h}\right), \quad \forall w_{h} \in V_{h}, i=1,2, \ldots, N, \\
& y_{h}^{0}=y_{0}^{h}(x), \quad x \in \Omega, \\
& \left(\frac{p_{h}^{i-1}-p_{h}^{i}}{\Delta t}, q_{h}\right)+a\left(q_{h}, p_{h}^{i-1}\right)+\left(\phi^{\prime}\left(y_{h}^{i}\right) p_{h}^{i-1}, q_{h}\right) \\
& =\left(y_{h}^{i}-y_{d}\left(x, t_{i}\right), q_{h}\right), \quad \forall q_{h} \in V_{h}, i=N, N-1, \ldots, 1, \\
& \quad p_{h}^{N}(x)=0, \quad x \in \Omega, \\
& \left(u_{h}^{i}+B^{*} p_{h}^{i-1}, v_{h}-u_{h}^{i}\right)_{U} \geq 0, \quad \forall v_{h} \in K_{h}, i=1,2, \ldots, N,
\end{aligned}
$$

where $y_{0}^{h} \in V^{h}$ is an approximation of $y_{0}$ defined above.

Example 5. The example is to solve the following 2D parabolic control problem:

$$
\begin{aligned}
& \min \quad \int_{0}^{1} \frac{1}{2}\left(\left\|y-y_{d}\right\|^{2}+\left\|u-u_{0}\right\|^{2}\right) d t \\
& \text { s.t. } \quad \frac{\partial y}{\partial t}-\Delta y+y^{3}=u+f, \quad u \geq 0
\end{aligned}
$$

where

$$
\begin{gathered}
y(x, t)=\sin \left(\pi x_{1}\right) \sin \left(\pi x_{2}\right) t, \\
u_{0}(x, t)=0.5-\sin \left(\pi x_{1}\right) \sin \left(\pi x_{2}\right) t, \\
y_{d}(x, t)=y+\frac{\partial p}{\partial t}+\Delta p-3 y^{2} p, \\
p(x, t)=\sin \left(\pi x_{1}\right) \sin \left(\pi x_{2}\right)(1-t), \\
u(x, t)=\max \left(u_{0}-p, 0\right), \\
f(x, t)=\frac{\partial y}{\partial t}-\Delta y+y^{3}-u .
\end{gathered}
$$

The dual equation of the state equation is

$$
-\frac{\partial p}{\partial t}-\Delta p+3 y^{2} p=y-y_{d}
$$

Table 1 shows the errors $\left\|u-u_{h}\right\|_{L^{2}\left(J ; L^{2}\right)}$ and $\| Q_{h} u-$ $u_{h} \|_{L^{2}\left(J ; L^{2}\right)}$ on a sequence of uniformly refined meshes, where $\left\|u-u_{h}\right\|_{L^{2}\left(j ; L^{2}\right)}$ denotes

$$
\left\|u-u_{h}\right\|_{L^{2}\left(J ; L^{2}\right)}=\left(\sum_{i=0}^{N}\left\|u\left(t_{i}\right)-u_{h}^{i}\right\|_{0}^{2} \Delta t\right)^{1 / 2}
$$

and similarly for $\left\|Q_{h} u-u_{h}\right\|_{L^{2}\left(J ; L^{2}\right)}$. We choose $\Delta t=0.005$ in our numerical example. The superconvergence phenomenon of $\left\|Q_{h} u-u_{h}\right\|_{L^{2}\left(J ; L^{2}\right)}$ can be observed clearly from Table 1. 
TABLE 1: The error of example on sequential uniform refined meshes.

\begin{tabular}{lcc}
\hline Resolution & $\left\|u-u_{h}\right\|_{L^{2}\left(J ; L^{2}\right)}$ & $\left\|Q_{h} u-u_{h}\right\|_{L^{2}\left(J ; L^{2}\right)}$ \\
\hline $10 \times 10$ & $4.373 E-2$ & $5.369 E-3$ \\
$20 \times 20$ & $2.180 E-2$ & $2.021 E-3$ \\
$40 \times 40$ & $1.096 E-3$ & $6.217 E-4$ \\
$80 \times 80$ & $5.483 E-4$ & $2.303 E-4$ \\
\hline
\end{tabular}

\section{Conclusion}

In this paper, we present the superconvergence analysis for the semidiscrete finite element approximation of optimal control problems governed by semilinear parabolic equations. Here, the results seem to be new and detailed proof can be used in more areas. We will study some results of superconvergence for optimal control, such as superconvergence for optimal control problems governed by semilinear parabolic equations with mixed finite element method.

\section{Conflict of Interests}

The authors declare that there is no conflict of interests regarding the publication of this paper.

\section{Acknowledgments}

This work is supported by the National Science Foundation of China (11271145), Foundation for Talent Introduction of Guangdong Provincial University, Specialized Research Fund for the Doctoral Program of Higher Education (20114407110009), the Project of Department of Education of Guangdong Province (2012KJCX0036), and TianYuan Special Funds of the National Natural Science Foundation of China (Grant no. 11226313).

\section{References}

[1] W. Alt, "On the approximation of infinite optimization problems with an application to optimal control problems," Applied Mathematics and Optimization, vol. 12, no. 1, pp. 15-27, 1984.

[2] W. Alt and U. Mackenroth, "Convergence of finite element approximations to state constrained convex parabolic boundary control problems," SIAM Journal on Control and Optimization, vol. 27, no. 4, pp. 718-736, 1989.

[3] Y. Chen, "Superconvergence of quadratic optimal control problems by triangular mixed finite element methods," International Journal for Numerical Methods in Engineering, vol. 75, no. 8, pp. 881-898, 2008.

[4] Y. Chen, "Superconvergence of mixed finite element methods for optimal control problems," Mathematics of Computation, vol. 77, no. 263, pp. 1269-1291, 2008.

[5] Y. Chen and Y. Dai, "Superconvergence for optimal control problems governed by semi-linear elliptic equations," Journal of Scientific Computing, vol. 39, no. 2, pp. 206-221, 2009.

[6] Y. Chen and W. Liu, "Error estimates and superconvergence of mixed finite element for quadratic optimal control," International Journal of Numerical Analysis and Modeling, vol. 3, no. 3, pp. 311-321, 2006.
[7] Y. Chen and W. Liu, "A posteriori error estimates for mixed finite element solutions of convex optimal control problems," Journal of Computational and Applied Mathematics, vol. 211, no. 1, pp. 76-89, 2008.

[8] D. A. French and J. T. King, "Approximation of an elliptic control problem by the finite element method," Numerical Functional Analysis and Optimization, vol. 12, no. 3-4, pp. 299-314, 1991.

[9] L. S. Hou and J. C. Turner, "Analysis and finite element approximation of an optimal control problem in electrochemistry with current density controls," Numerische Mathematik, vol. 71, no. 3, pp. 289-315, 1995.

[10] G. Knowles, "Finite element approximation of parabolic time optimal control problems," Society for Industrial and Applied Mathematics, vol. 20, no. 3, pp. 414-427, 1982.

[11] I. Lasiecka, "Ritz-Galerkin approximation of the time optimal boundary control problem for parabolic systems with Dirichlet boundary conditions," SIAM Journal on Control and Optimization, vol. 22, no. 3, pp. 477-500, 1984.

[12] R. S. McKnight and W. E. Bosarge, Jr., "The Ritz-Galerkin procedure for parabolic control problems," SIAM Journal on Control and Optimization, vol. 11, pp. 510-524, 1973.

[13] D. Meidner and B. Vexler, "A priori error estimates for spacetime finite element discretization of parabolic optimal control problems. I. Problems without control constraints," SIAM Journal on Control and Optimization, vol. 47, no. 3, pp. 1150-1177, 2008.

[14] D. Meidner and B. Vexler, "A priori error estimates for spacetime finite element discretization of parabolic optimal control problems. II. Problems with control constraints," SIAM Journal on Control and Optimization, vol. 47, no. 3, pp. 1301-1329, 2008.

[15] P. G. Ciarlet, The Finite Element Method for Elliptic Problems, North-Holland Publishing, Amsterdam, The Netherlands, 1978.

[16] J. Haslinger and P. Neittaanmäki, Finite Element Approximation for Optimal Shape Design, John Wiley \& Sons, Chichester, UK, 1988.

[17] P. Neittaanmäki and D. Tiba, Optimal control of Nonlinear Parabolic Systems, vol. 179, Marcel Dekker, New York, NY, USA, 1994.

[18] D. Tiba, Lectures on the Optimal Control of Elliptic Equations, University of Jyvaskyla Press, Jyvaskyla, Finland, 1995.

[19] Q. Li and H. Du, "Lp error estimates and superconvergence for finite element approximation for nonlinear parabolic problems," Journal of the Korean Society for Industrial and Applied Mathematics, vol. 4, pp. 67-77, 2000.

[20] C. Hou, Y. Chen, and Z. Lu, "Superconvergence property of finite element methods for parabolic optimal control problems," Journal of Industrial and Management Optimization, vol. 7, no. 4, pp. 927-945, 2011.

[21] K. Chrysafinos, M. D. Gunzburger, and L. S. Hou, "Semidiscrete approximations of optimal Robin boundary control problems constrained by semilinear parabolic PDE," Journal of Mathematical Analysis and Applications, vol. 323, no. 2, pp. 891-912, 2006.

[22] F. Tröltzsch, "Semidiscrete Ritz-Galerkin approximation of nonlinear parabolic boundary control problems-strong convergence of optimal controls," Applied Mathematics and Optimization, vol. 29, no. 3, pp. 309-329, 1994.

[23] J. L. Lions and E. Magenes, Non Homogeneous Boundary Value Problems and Applications, vol. 181, Edited by B. Grandlehre, Springer, 1972. 
[24] J.-L. Lions, Optimal Control of Systems Governed by Partial Differential Equations, Springer, New York, NY, USA, 1971.

[25] V. Girault and P.-A. Raviart, Finite Element Methods for NavierStokes Equations, vol. 5, Springer, Berlin, Germany, 1986.

[26] R. Li, W. Liu, and N. Yan, "A posteriori error estimates of recovery type for distributed convex optimal control problems," Journal of Scientific Computing, vol. 33, no. 2, pp. 155-182, 2007.

[27] W. Liu and D. Tiba, "Error estimates in the approximation of optimization problems governed by nonlinear operators," Numerical Functional Analysis and Optimization, vol. 22, no. 78, pp. 953-972, 2001.

[28] Y. Huang, R. Li, W. Liu, and N. Yan, "Adaptivemulti-mesh finite element approximation for constrained optimal control problems," SIAM Journal on Control and Optimization. In press.

[29] R. Li and W. B. Liu, http://dsec.pku.edu.cn/ rli/software .php\#AFEPack. 


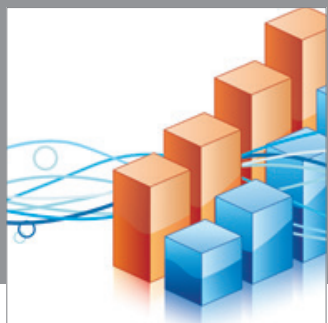

Advances in

Operations Research

mansans

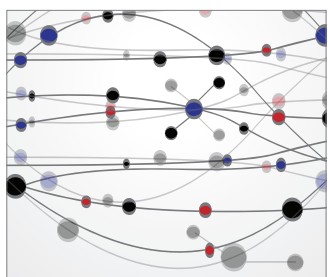

The Scientific World Journal
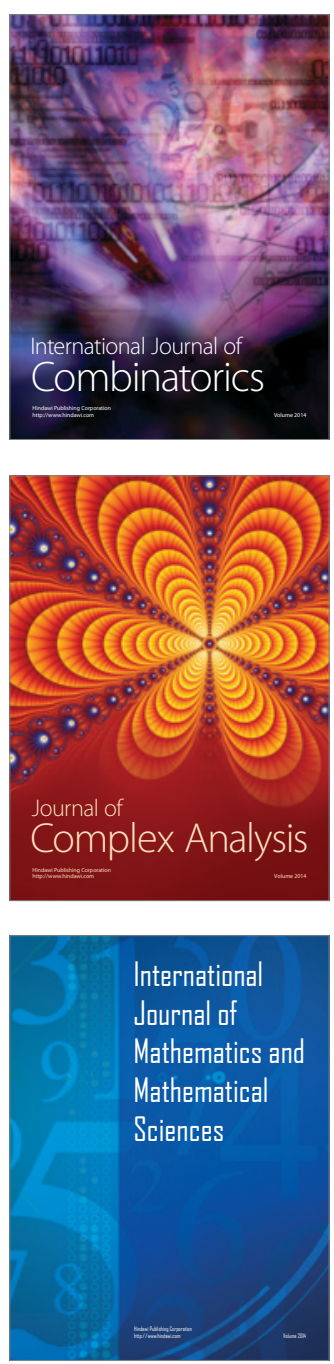
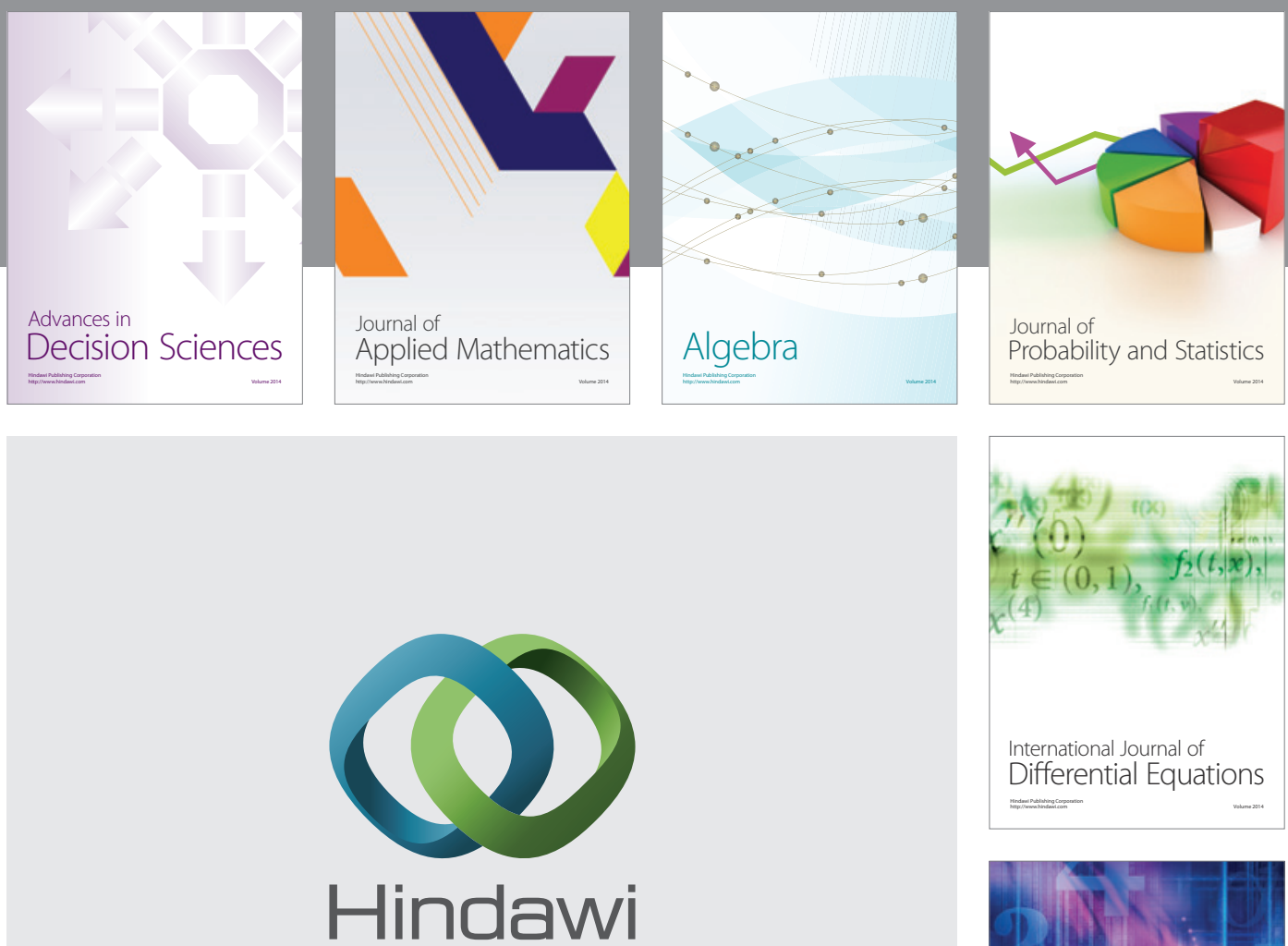

Submit your manuscripts at http://www.hindawi.com
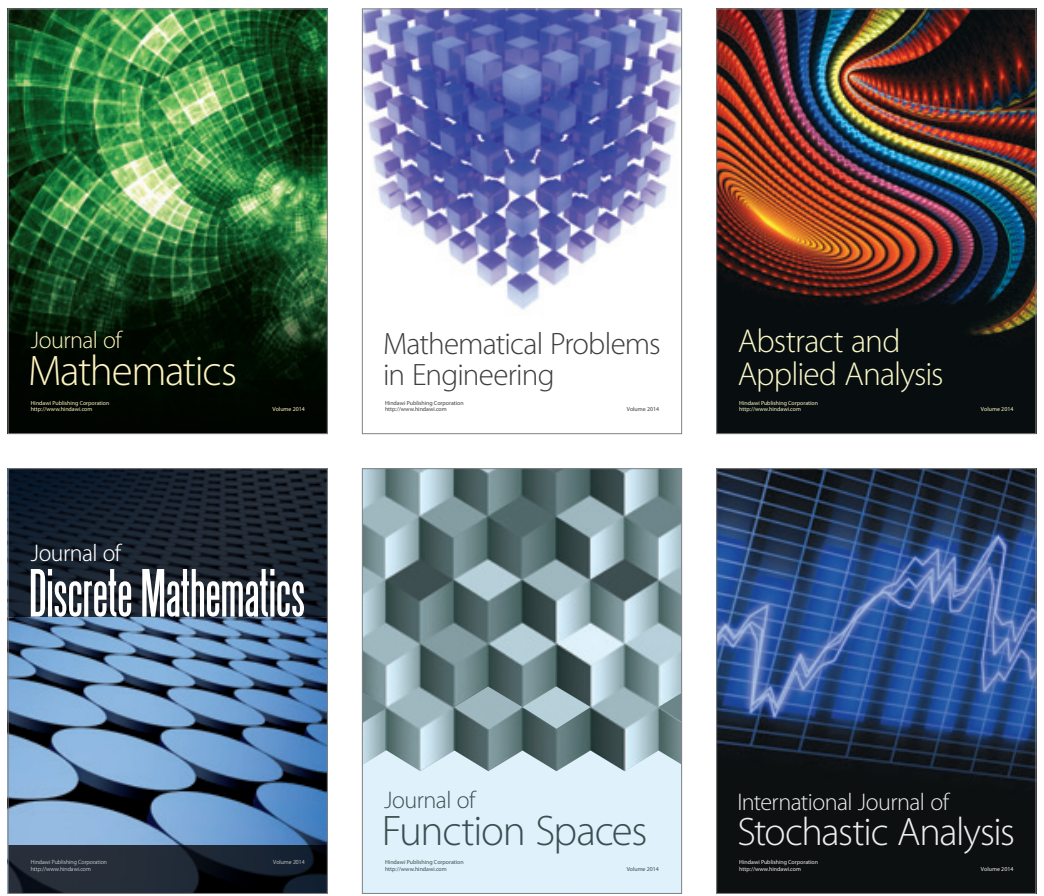

Journal of

Function Spaces

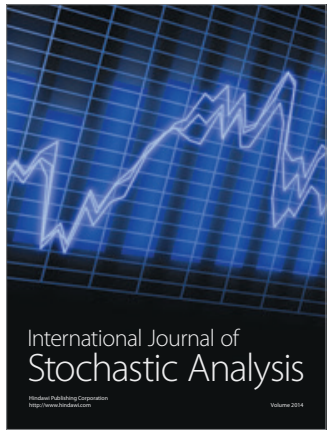

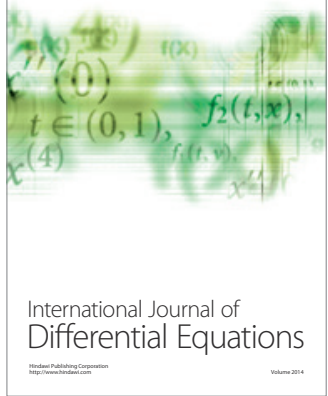
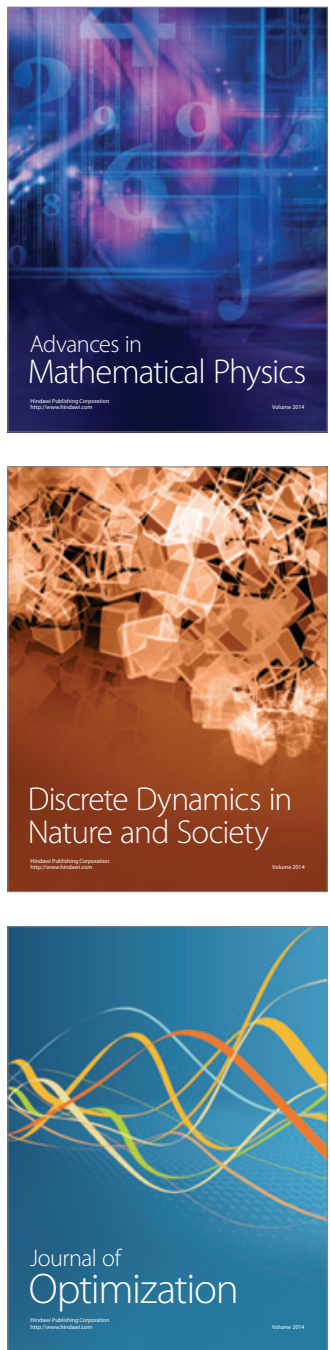